\title{
Économie et droit du travail
}

Des discours concurrents mais non rivaux

Economics and labor law: competing but not rival approaches

\section{Cécile Bourreau-Dubois et Bruno Deffains}

\section{(2) OpenEdition}

\section{Journals}

Édition électronique

URL : http://journals.openedition.org/travailemploi/1631

DOI : 10.4000/travailemploi.1631

ISSN : 1775-416X

Éditeur

DARES - Ministère du Travail

Édition imprimée

Date de publication : 15 décembre 2009

Pagination : 09-16

ISSN : 0224-4365

Référence électronique

Cécile Bourreau-Dubois et Bruno Deffains, «Économie et droit du travail », Travail et Emploi [En ligne], 120 | octobre-décembre 2009, mis en ligne le 30 décembre 2011, consulté le 19 avril 2019. URL :

http://journals.openedition.org/travailemploi/1631; DOI : 10.4000/travailemploi.1631 


\title{
Économie et droit du travail: des discours concurrents mais non rivaux
}

\author{
Cécile Bourreau-Dubois $\left({ }^{*}\right)$, Bruno Deffains $(* *)$
}

\begin{abstract}
Cet article défend la thèse selon laquelle l'analyse économique du droit est une voie, parmi d'autres, pour établir un dialogue entre l'approche économique et l'approche juridique en matière d'analyse du droit du travail. En premier lieu, sont présentées les raisons pour lesquelles il est légitime de faire de l'analyse économique du droit du travail, tout en soulignant la nécessité - pour que cette analyse soit pertinente - d'étudier finement la façon dont les règles de droit du travail influencent le comportement des acteurs. En second lieu, les auteurs illustrent les apports de l'analyse économique du droit du travail en examinant - d'un point de vue tant théorique que empirique - la validité de l'hypothèse de substitution, hypothèse qui est généralement mobilisée pour rendre compte de l'évolution contrastée des licenciements pour motif économique et des licenciements pour motif personnel depuis les années 1990.
\end{abstract}

Les pays industrialisés ont fait depuis longtemps le choix d'une régulation du marché du travail par le droit. S'agissant des conditions de travail, de la rémunération des salariés ou encore des procédures de licenciement, l'encadrement juridique est omniprésent et les tribunaux sont bien souvent au cœur des débats relatifs à la mise en œuvre du contrat de travail. Par ailleurs, le droit du travail et sa mise en œuvre sont souvent perçus comme un moyen de rééquilibrer les rapports de force entre employeur et salarié, ce dernier apparaissant en situation d'infériorité au moment de la négociation du contrat de travail. Parallèlement, la théorie économique s'est souvent attachée à caractériser les meilleurs contrats possibles entre l'employeur (le principal) et le salarié (l'agent) lorsque ce dernier possède une meilleure information sur les éléments pertinents pour le contrat. En pratique, s'il est difficile d'observer l'effort de l'agent, des mécanismes doivent être trouvés pour l'inciter à entreprendre des actions conformes aux intérêts du principal. Quelle est la place du droit dans un tel contexte? À vrai dire, la théorie économique ne s'en est guère préoccupée. En général, elle se limite à envisager l'intervention du tribunal afin de compléter ex post le contrat lorsqu'il est impossible pour le principal de vérifier les performances de l'agent. Le dialogue entre économistes et juristes peut donc apparaître d'emblée délicat dans la mesure où leur manière d'appréhender le contrat de travail diffère sensiblement. L'un des apports de l'économie du droit est justement de permettre de dépasser ce clivage et de faire dialoguer les deux approches, au bénéfice des deux disciplines. Il existe plusieurs manières de nouer des liens entre le droit et la science économique. L'analyse économique du

(*) BETA, université Nancy 2. Cecile-dubois@univ-nancy2.fr (**) EconomiX, université Paris Ouest Nanterre La Défense. bruno.deffains@univ-nancy2.fr droit, qui est la perspective interdisciplinaire sans doute la plus connue et la plus répandue, constitue un point d'entrée parmi d'autres(1).

En faisant appel à des concepts empruntés à la science économique, l'analyse économique du droit cherche le plus souvent à mettre au jour la rationalité économique(2) sous-jacente des règles juridiques et les principaux effets prévisibles de leur changement. Dans ce cadre théorique, les règles juridiques sont vues comme des mécanismes incitatifs par lesquels le droit va influencer le comportement des agents économiques, qui vont réagir aux incitations du système juridique. L'analyse économique du droit prétend donc fournir des éléments pour un jugement éclairé sur les institutions juridiques et sur les réformes proposées. Les travaux entrepris dans cette perspective ont souvent eu l'effet d'un déclic sur le monde juridique, suscitant un vaste mouvement d'exploration pour découvrir comment ces nouvelles idées pouvaient s'appliquer aux règles juridiques dans les différents champs du droit. Les recherches touchant au droit de la responsabilité ou aux procédures de règlement des conflits fournissent de nombreux exemples de ce type de travaux (DefFains, 1997, 2001). S'il y a un relatif consensus sur cette façon de concevoir l'analyse économique du droit, il convient toutefois de noter que les démarches poursuivies par les différents travaux n'ont pas tous la même ambition. Il nous semble important de distinguer les travaux qui s'inscrivent dans une logique juridique purement «interne» de

(1) Sur le pluralisme des approches entre droit et économie, voir par exemple DefFAINS, FEREY (2007). Les perspectives institutionnelles, autrichiennes, comportementales et néoclassiques sont notamment évoquées dans cet article.

(2) Soulignons ici qu'il ne s'agit pas pour l'analyse économique du droit de nier l'existence d'autres types de rationalité des règles de droit (juridique, sociologique, etc.). 
ceux qui adoptent un point de vue «externe». Dans la première catégorie, le point de départ est un problème juridique et le savoir économique est utilisé pour élaborer ou pour appliquer une règle juridique. C'est par exemple le cas de Posner, qui a toujours affiché l'ambition de démontrer de quelle manière les outils de l'analyse micro-économique peuvent être mobilisés pour identifier des règles de droit plus efficaces. Dans la seconde catégorie, nous pouvons ranger tous les travaux dont l'ambition première est l'étude de problèmes de nature avant tout économique, telles que l'allocation des ressources rares ou la correction des défaillances des marchés. Il est clair que ni Coase sur les externalités, ni Becker à propos de la politique pénale n'ont jamais prétendu faire du droit; ils se sont intéressés au droit dans la mesure où ils le conçoivent comme un moyen de réguler les comportements et les marchés. Par extension, il nous semble que la logique dans laquelle doit s'inscrire l'analyse économique $\mathrm{du}$ droit du travail est avant tout une logique externe. C'est à la question de l'efficacité de la régulation du marché du travail par le droit qu'il convient d'apporter une réponse. Ce regard externe sur le droit du travail nous semble légitime dans le sens où le système juridique n'est pas isolé du reste de la société. Ainsi, par exemple, on ne peut nier le fait que les règles de droit ont une incidence sur le comportement des agents économiques, voire même que les acteurs du droit sont influencés par le contexte économique et social qui les entourent(3). Bien entendu, cette interdépendance ne remet pas en cause la légitimité de l'analyse juridique du droit du travail.

La thèse que l'on cherche ici à défendre est que l'approche juridique et l'approche économique du droit $\mathrm{du}$ travail doivent non seulement pouvoir coexister mais également dialoguer. En premier lieu, il s'agit de faire valoir la possibilité de l'existence d'une variété de points de vue sur les règles de droit, en particulier de l'existence de discours «concurrents» au discours de la doctrine juridique sur le droit positif, que ce soit une analyse économique normative et/ou analyse économique positive. En second lieu, ces discours ne doivent pas être considérés comme rivaux mais comme complémentaires. En effet, l'analyse économique du droit du travail ne peut être menée à bien que si elle considère sérieusement le droit, tant dans son contenu que dans sa mise en œuvre par les tribu-

(3) CHINo et al. (2003) analysent les jugements rendus par les juges du travail dans le contexte de litiges nés à la suite du licenciement pour motif personnel d'un salarié et les mettent en parallèle avec les caractéristiques du marché du travail. À partir de données italiennes, les auteurs étudient le lien entre le taux de chômage dans l'économie et les décisions prises par les juges dans des affaires de licenciement pour faute. L'étude montre que lorsque le taux de chômage est élevé, les juges ont plutôt tendance à favoriser les salariés. Ainsi, une même faute commise par un salarié pourrait légitimer un licenciement lorsque le taux de chômage est faible et ne serait pas condamnable avec un taux de chômage élevé. Cette analyse montre que les conditions macro-économiques locales constituent l'un des critères de décision des juges du travail. naux. En particulier, le juge ne saurait être appréhendé comme un simple régulateur dans la mesure où les conditions de son intervention sont différentes de celles du régulateur public. Pour cette raison, un dialogue avec les juristes est nécessaire. Inversement, on peut espérer que les juristes trouvent également de l'intérêt dans la prise en considération de la méthode économique et intègrent quelques conclusions ou méthodes dans leur façon d'appréhender et de pratiquer le droit. La question posée est simplement de savoir en quoi l'analyse économique du droit peut contribuer à ce que le droit soit bien fait. Dès lors que les règles de droit agissent sur les comportements en changeant les incitations qui gouvernent les décisions des individus, il semble possible d'appréhender les dispositifs juridiques comme un moyen d'agir sur les préférences individuelles. Cette perspective n'est d'ailleurs pas sans rappeler la position de Durkheim sur la division du travail social(4). Notre démarche se veut toutefois plus pragmatique puisqu'elle vise à montrer pourquoi les juristes du travail peuvent avoir intérêt, indépendamment de l'avantage de développer leurs connaissances et leurs horizons, à s'ouvrir aux raisonnements mobilisés par l'analyse économique.

Le plan de l'article suivra la logique suivante (5). Dans un premier temps il s'agit de revenir sur les justifications de faire de l'analyse économique du droit du travail. Dans un deuxième temps, les perspectives qu'offre le développement d'une telle analyse sont présentées.

\section{Pourquoi une analyse économique du droit du travail?}

Les institutions du marché du travail jouent un rôle essentiel dans la distribution des pouvoirs de négociation des employeurs et des salariés. Or, ces pouvoirs de négociations affectent, entre autres choses, le résultat des négociations collectives, le taux de chômage ou encore l'efficacité des mécanismes incitatifs qui caractérisent les contrats de travail. Des institutions différentes produisent des effets variés sur l'ensemble de ces éléments. Par exemple, les dispositifs en matière de protection

(4) Dans sa célèbre thèse publiée en 1893 sur le lien social, Durkheim explique qu'avec la complexité croissante de la société, les individus se différencient de plus en plus, la division du travail progresse et ce, dans toutes les sphères de la vie sociale (économie, administration, justice, science, etc.). Or, la spécialisation, la différenciation accrue des individus entre eux les rend de facto interdépendants. La division du travail est en réalité source de solidarité sociale, de cohésion sociale: dans le même temps qu'elle différencie les individus, elle les rend complémentaires et c'est pourquoi, selon Durkheim, elle contraint les individus à vivre ensemble.

(5) Les réflexions développées dans cet article sont inspirées du rapport réalisé sous la responsabilité des auteurs et remis à la DARES en mars 2008 sous l'intitulé «Analyse économique du droit du travail». 
sociale, les règles du droit du licenciement ou celles qui régissent les négociations collectives vont agir directement sur la capacité des salariés à extraire une fraction plus ou moins importante des gains issus de la relation de travail.

C'est de cette manière que les économistes abordent, le plus souvent, le droit du travail. La relation de travail est appréhendée comme un contrat incomplet compte tenu des asymétries informationnelles qui la caractérise. Le droit du travail apparaît alors comme un moyen de compléter le contrat, notamment à travers l'intervention du juge en cas de désaccord entre les parties. L'optique est celle de l'organisation industrielle qui voit la firme comme un nœud de contrats. Plus généralement, la démarche en économie du travail consiste souvent à appréhender les relations de travail au travers des conflits entre les salariés et les employeurs. Cela détermine la manière de concevoir le rôle du droit puisqu'il contribue à déterminer les positions respectives des parties dans la négociation salariale. Cela n'est d'ailleurs pas sans rappeler les travaux des spécialistes de finance d'entreprise, qui appréhendent le gouvernement d'entreprise comme une divergence d'intérêt entre les dirigeants et les actionnaires. Ici aussi, le droit est un instrument permettant de déterminer les pouvoirs de négociation des deux parties. Par ce biais, le droit affecte l'efficacité de la relation contractuelle.

On peut noter ici que les deux types de «conflits» sont présents simultanément et interagissent dans l'entreprise. On peut donc s'étonner du peu d'importance accordée à ce jour aux règles du droit du travail par les économistes. Les dernières années ont pourtant été marquées par un essor des travaux qui font de la qualité des institutions juridiques une condition sine qua non du développement économique. Il s'agit notamment des travaux de la $\mathrm{New}$ Comparative Economics (LA PORTA, LOPEZ DE Silanes, Shleifer, 2008). De nature empirique, ces recherches ont notamment servi de fondements à la mise en place du programme Doing Business de la Banque mondiale. Elles soutiennent l'existence d'une corrélation entre appartenance à un système juridique et développement économique, en privilégiant le droit de Common law. Plus précisément, ces travaux s'efforcent de mettre en évidence les effets de l'origine juridique sur les performances des systèmes financiers dans les pays de l'OCDE. Ils montrent que l'origine du système juridique (anglo-saxon, français, allemand ou scandinave), le contenu des règles et leurs conditions d'application influencent le degré de protection accordée aux investisseurs ainsi que le niveau de développement des marchés financiers.

Ces études comparatives décrivent une situation dans laquelle les pays de Common law offriraient une meilleure protection aux investisseurs que les pays de droit civil. Cette particularité expliquerait pourquoi les pays anglo-saxons ont des marchés financiers plus développés, une propriété du capital plus dispersée et des capitaux propres plus importants que ceux relevant de la tradition civiliste. Il ressort de ces analyses qu'en assurant une protection juridique supérieure, le système de Common law dominerait le(s) système(s) de droit civil. L'actionnariat dispersé et le système de gouvernement d'entreprise fondé sur les marchés seraient «préférables» à un système où l'actionnariat est concentré et où le gouvernement d'entreprise repose sur des blocs de contrôle. L'explication résiderait dans le fait que la dispersion de l'actionnariat irait de pair avec des marchés financiers développés. $\mathrm{La}$ Banque mondiale dans le cadre du programme Doing Business a repris à son compte cette approche. Sont ainsi passés au crible la plupart des domaines du droit qui influencent la vie de l'entreprise (droit financier, droit commercial, droit des faillites...). On peut noter que la question des modalités de résolution des conflits au sein de l'entreprise, même si elle n'est pas au cœur de la discussion, n'échappe pas totalement à l'évaluation. Des études ont en particulier cherché à intégrer le degré de formalisme des procédures (DJANKov et al., 2004). Toutefois, la question du fonctionnement de la justice en matière économique, notamment lorsqu'elle touche le contentieux du travail, est largement ignorée. De ce fait, les conclusions tirées de ces recherches apparaissent manifestement biaisées (ARRUNADA, 2007).

L'intérêt principal de ces travaux est d'envisager les relations droit-économie sous un nouveau jour mettant en évidence l'importance de la régulation juridique pour une organisation efficace des activités économiques. Toutefois, la régulation juridique ne saurait se limiter aux relations financières. Il est essentiel de considérer l'ensemble des parties impliquées dans le fonctionnement de l'entreprise, en particulier à travers les relations de travail. Un certain nombre de travaux ont déjà mis en avant la complémentarité des stratégies entre salariés et dirigeants dans le cas des tentatives de prise de contrôle (Hellwig, 2000; Pagano, Volpin, 2004). Plus généralement, des études récentes (RoE, 2008) indiquent clairement que plus que la protection des actionnaires, ce sont les règles de protection des salariés qui expliquent le mieux les choix des entreprises en matière de structure de capital et de concentration de la propriété. Autant de raisons de considérer le droit du travail.

C'est en partie dans cette perspective que s'inscrivent les travaux qui entendent mettre en évidence l'inadéquation du droit du travail aux mutations du marché du travail (De Virville, 2004, CAmbdessus, 2004, Cahuc, Kramarz, 2004, Blanchard, Tirole, 2003). Ainsi, l'intervention du juge dans le cas des licenciements est souvent stigmatisée, en particulier dans le cas des licenciements pour motif économique où le juge ne saurait intervenir efficacement faute de culture économique. D'où la proposition de substituer au système existant un mécanisme d'incitation financière en dehors de toute intervention du juge. De même, est visée la législation sur les licencie- 
ments. Traditionnellement, on ne peut licencier que pour deux raisons : pour motif économique ou pour motif personnel. Cette situation créerait une zone de non-droit au sens où l'entreprise qui constaterait une incompatibilité entre un salarié et son emploi pourrait être incitée à le harceler pour le faire démissionner ou pour le pousser à la faute afin d'avoir une bonne raison de le licencier (WASMER, 2006). Ne vaudrait-il pas mieux alors permettre ce licenciement en tant que tel, en l'encadrant, sous un régime d'indemnité plus favorable, par exemple, que les autres ruptures? C'est le sens des nouveaux dispositifs visant à créer un troisième type de rupture du contrat de travail, la séparation à l'amiable. Enfin, dernier exemple, l'incertitude juridique du licenciement - liée notamment à la possibilité pour le salarié de contester son licenciement aux prud'hommes - représente un coût pour les entreprises sans être pour autant un bénéfice pour les salariés. De nombreuses propositions insistent sur le fait qu'il faudrait transformer cette insécurité juridique en élément monétaire. Elle serait chiffrée et transformée en indemnité supplémentaire pour le salarié en contrepartie d'une sécurité pour l'employeur (CAHUC, KRAmarz, 2004).

À défaut de démonstrations probantes, l'idée est donc bien ancrée selon laquelle le droit du travail pèserait lourdement sur les décisions des acteurs économiques. Or dans la plupart de ces études, la remise en cause du droit du travail - et plus particulièrement celle de l'intervention du juge - reste extrêmement générale dans la mesure où elles s'intéressent assez peu à la manière dont les acteurs économiques peuvent se saisir du droit du travail ou à la manière dont les juges jouent effectivement leur rôle. C'est donc bien avec une volonté affirmée de dépasser cette vision relativement superficielle du droit du travail et de son usage par les acteurs que les recherches à l'intersection de l'économie et du droit du travail doivent être entreprises afin de poser quelques jalons dans le champ d'une véritable analyse économique du droit du travail. Il importe d'étudier particulièrement la façon dont les règles de droit du travail et le système judiciaire influencent la relation de travail, et en particulier le moment de la rupture du contrat de travail. Pour cela, il convient d'analyser comment les employeurs et les salariés se saisissent des règles du droit du travail dans leur comportement économique(6).

(6) Une telle démarche conduit donc à s'écarter du clivage habituel qui tend à opposer d'une part les arguments des économistes du travail - plutôt favorables à l'idée de flexibilité - et ceux des juristes du travail d'autre part - plutôt favorable à la sécurité et à la protection de la partie «faible». Certains concepts à la mode tendent d'ailleurs de concilier les points de vue, à travers la notion de «flexicurité» en particulier. Ces travaux sont utiles mais ils ne répondent pas vraiment à la question de fond posée à l'économiste par les règles du droit du travail, à savoir la façon dont ces règles de droit vont être perçues par les agents économiques et comment ces derniers vont s'en saisir pour s'y adapter.
La démarche proposée n'a rien de surprenant et la boîte à outils de l'économiste est suffisamment riche pour mener à bien des travaux théorique et empirique. Il est étonnant d'observer que cette démarche n'a pas été entreprise jusqu'à présent. Les raisons en sont multiples. Le cloisonnement disciplinaire n'explique pas tout. L'analyse économique du droit s'est développée surtout dans le système de la Common law nord américaine et force est de constater que, dans cet environnement, la question de l'encadrement juridique des relations de travail n'a pas suscité beaucoup de recherches. Dans le même temps, on a pu penser parfois que les outils proposés par l'analyse économique du droit étaient réservés à cette tradition juridique, héritée de la philosophie réaliste, et ne pouvaient (devaient?) pas s'appliquer aux systèmes de tradition civiliste. Fort heureusement, cette vision étriquée des relations droit-économie a fait long feu. Parallèlement, les économistes n'ont sans doute pas suffisamment fait d'efforts pour comprendre les mécanismes juridiques. Ces derniers leur semblent le plus souvent inutilement complexes et le discours qui les accompagnent pratiquement insaisissables, compromettant par là même les possibilités de réception de l'analyse économique par le droit du travail. Figure emblématique de ce type de difficultés, le juge. Dans un univers de contrats incomplets, le recours au tribunal apparait le plus souvent comme un moyen de compléter le contrat de travail. Dès lors, si les contrats pouvaient être simplifiés et définis de manière plus complète, on devrait pouvoir se passer le plus souvent du recours au juge. C'est certainement oublier que le rôle du juge ne saurait se cantonner à cette fonction de complétude de la relation contractuelle. Il ne peut être assimilé à un simple régulateur.

\section{Comment faire de l'analyse économique du droit du travail?}

Le recours à l'analyse économique pour évaluer les effets du droit du travail sur les relations du travail, sur la gestion de la main-d'œuvre ou sur le fonctionnement du marché du travail doit permettre de faire émerger, par la mobilisation de différentes méthodologies, des hypothèses inédites, susceptibles d'être (in)validées empiriquement. C'est ce que nous allons essayer ici d'illustrer en nous appuyant sur un fait stylisé, très connu des économistes: l'évolution contrastée depuis les années 1990 des licenciements pour motif économique (LME) et des licenciements pour motif personnel (LMP).

On assiste, depuis les années 1990 à une baisse constante du recours au licenciement économique et une hausse des licenciements pour motifs person- 
Graphique 1. Évolution des licenciements entre 1997 et 2009

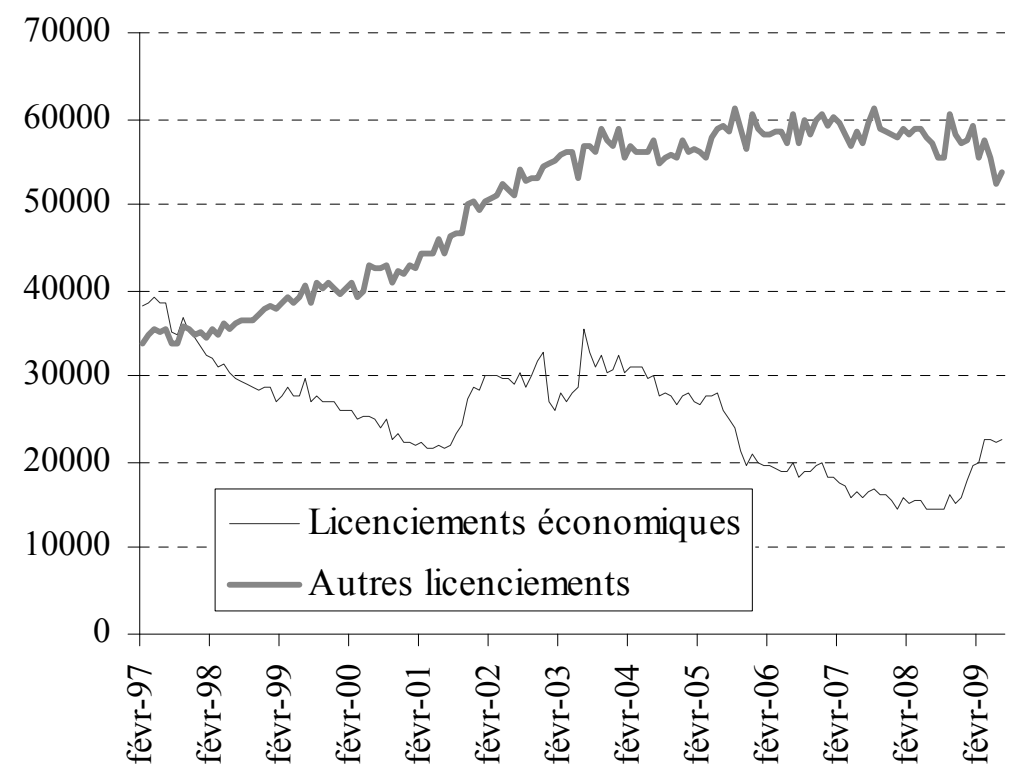

Champ : demandeurs d'emplois inscrits à Pôle Emploi en catégories A,B et C, France métropolitaine.

Source: STMT-Pôle Emploi, Dares.

nels (voir graphique 1) (7). En 2003, les LMP sont presque trois fois plus nombreux que les LME (Lagarenne, Le Roux, 2006). Cette situation traduit une lente mais constante érosion des licenciements économiques après une hausse importante en 1993 (près de 600000 par an), les licenciements économiques n'ont cessé de baisser (excepté en 2002) pour se situer autour de 200000 par an en 20032004. Ainsi, en moins de dix ans, les licenciements économiques ont été divisés par trois. Même s'il est difficile de connaître de manière précise la ventilation entre licenciements individuels et licenciements collectifs, les données disponibles pour les établissements de cinquante salariés ou plus incitent à penser que cette évolution tient aussi bien à une baisse des licenciements économiques individuels que collectifs. Parallèlement, les licenciements pour motif personnel n'ont, eux, cessé d'augmenter et sont passés d'environ 380000 en 1993 à 560000 en 2003 (Munoz-Perez, Serverin, 2005). Cette analyse

(7) Rappelons que le droit du travail français reconnait deux sortes de licenciements. L'un pour motif personnel nécessite une «cause réelle et sérieuse»: il en est ainsi en cas de «faute constituant une cause réelle et sérieuse» (réitération de fautes légères par exemple) ou de faute grave ou encore de faute lourde (intention de nuire); l'autre pour motif économique (article L. 321-1 modifié loi 2005) il s'agit alors d'«un ou plusieurs motifs non inhérents à la personne du salarié, résultant d'une suppression ou d'une transformation d'emploi ou d'une modification refusée par le salarié d'un élément essentiel de son contrat de travail, consécutives notamment à des difficultés économiques ou à des mutations technologiques». Le notamment est important puisqu'il laisse la porte ouverte à un travail jurisprudentiel. Ainsi le 18 janvier 2006, la chambre sociale de la Cour de cassation a renversé sa jurisprudence en affirmant que des difficultés financières non immédiates peuvent constituer néanmoins une cause réelle et sérieuse de licenciements économiques. en nombre absolu nécessiterait d'être affinée considérablement mais elle est confirmée par l'évolution des taux de sortie. En effet, les taux de LME diminuent entre 1996 et 2001 de manière continue pour tous les secteurs et pour toutes les tailles d'entreprises, alors que les taux de sortie pour les LMP augmentent sur la même période (RICHET-MASTAING, 2003, p. 83).

Les explications de cette évolution contrastée des LME et des LMP sont dominées, dans le débat économique, par l'analyse en termes de substitution (CAhuc, Kramarz, 2004, p. 150): le coût judiciaire associé au LME inciterait les acteurs à des stratégies de contournement de la loi, dont le résultat serait de déguiser des licenciements pour motif économique en licenciement pour motif personnel. L'évolution de la structure des licenciements s'expliquerait donc par un usage stratégique du droit par les entreprises. Cependant, au-delà de la reconnaissance de l'importance de ce phénomène, la littérature économique fournit peu d'explications convaincantes (8). L'analyse économique du droit du travail telle que nous l'entendons conduit à dépasser l'intuition d'un usage stratégique du droit du travail pour en éprouver la robustesse théorique comme empirique. Pour ce faire, plusieurs pistes de recherche peuvent être explorées. Nous proposons ici de rendre compte de certaines d'entre elles.

(8) Cahuc et Kramarz se fondent sur des opinions de chefs d'entreprises, d'avocats ou de délégués du personnel (CAHUC, Kramarz, 2004) et Kramarz et Michaud se fondent sur l'estimation de la structure des coûts associés à la rupture de contrat (Kramarz, Michaud, 2005). 
En premier lieu, lorsqu'on s'intéresse aux entreprises qui licencient, on observe que les deux types de licenciement ne se rencontrent pas exactement dans les mêmes entreprises et ne concernent pas les mêmes types de salariés. Ainsi, les LME se concentrent sur les salariés travaillant dans des entreprises de petite taille (dix à quarante-neuf salariés) (Tomasini, Le Roux, 2002). Inversement, la proportion d'établissements recourant aux LME s'accroît avec la taille de l'établissement. Ainsi, en 2000, par exemple, $22 \%$ des entreprises de plus de 500 salariés ont eu recours au licenciement économique contre seulement $11 \%$ pour les entreprises de dix à quarante-neuf salariés (TomAsInI, Le RouX, 2002). Si pour l'ensemble des établissements d'au moins dix salariés du secteur privé industriel et commercial, le taux de LME est plus de deux fois moins élevé que celui de LMP $(0,7 \%$, contre $1,9 \%$ ), cette différence est bien plus marquée dans le domaine de la construction et du secteur tertiaire (respectivement, $0,4 \%$, contre $2,0 \%$ et $0,5 \%$ contre $2,1 \%)$. En revanche, les taux sont plus proches dans l'industrie $(1,0 \%$ contre $1,5 \%$ ) (ibidem). Plus précisément, c'est dans les secteurs traditionnels en difficulté que le taux de LME est le plus élevé (3,0\% dans l'habillement et le cuir, 2,2\% dans l'industrie textile), tandis que le taux de LMP est plus élevé dans les services aux entreprises $(2,8 \%)$ et le commerce $(2,6 \%)$ (ibidem). De même, on observe de fortes disparités entre les catégories socioprofessionnelles. Ainsi, les LME touchent davantage les ouvriers (près de $40 \%$ des personnes licenciées pour motif économique en 2000, alors qu'ils représentent un tiers des actifs occupés) et les salariés les plus âgés (ibidem). De tels constats empiriques, qui mettent en évidence l'existence d'un profil spécifique des entreprises licenciant pour raisons économiques, conduisent à mettre en cause la pertinence de l'hypothèse implicite faite par les promoteurs de la thèse de substitution, à savoir celle d'une substituabilité entre un licenciement personnel et un licenciement économique.

Une deuxième raison pourrait conduire à douter de la pertinence de la thèse de la substitution. En effet, l'analyse des taux de recours met en évidence que, depuis une dizaine d'années, le licenciement économique est extrêmement peu risqué dans la mesure où la probabilité que le licenciement soit contesté est extrêmement faible alors qu'inversement la probabilité d'un licenciement pour motif personnel soit contesté est, elle, relativement forte (9). L'hypothèse de substitution présenterait donc un aspect assez paradoxal puisque les employeurs auraient alors un comportement peu rationnel en substituant des licenciements risqués

(9) En 2003, le taux de recours en matière de licenciement économique était de $2,6 \%$ contre $26 \%$ pour les autres types de licenciements (MuNOZ-PEREZ, SERVERIN 2005). à des licenciements peu risqués. Pour certains, un tel paradoxe conduirait à invalider la thèse de la substitution. L'analyse économique du droit montre que cet argument en termes de risque judiciaire ne suffit pas cependant à se débarrasser de l'hypothèse de substitution. En utilisant les outils d'analyse du risque et de la théorie de la décision, on peut montrer que pour avoir une juste appréciation du risque judiciaire encouru par l'entreprise qui licencie, il faut tenir compte de trois paramètres: les coûts associés aux procédures; les taux de recours déposés devant les tribunaux et les taux d'acceptation des demandes. Ce faisant, l'analyse de la structure des incitations révèle que le LMP n'est pas, pour les entreprises, une solution intrinsèquement plus risquée que le LME. Dès lors, seule l'introduction de la psychologie du risque permet de déterminer les choix qui sont rationnels pour les acteurs en matière de type de licenciement. Plus précisément, on peut montrer que la neutralité au risque, la riscophilie et une aversion forte pour le risque vont dans le sens d'une préférence pour le motif économique; en revanche, une aversion au risque modérée fait pencher la décision en faveur du motif personnel. Certes ce type d'analyse ne permet pas de trancher de manière définitive en faveur ou non de l'interprétation en termes de substitution. Cependant, elle a pour intérêt de mettre en évidence que plus que les caractéristiques du droit du travail et la pratique des tribunaux c'est la subjectivité des acteurs dans un contexte de décision risqué qui importe pour analyser les effets du droit sur les comportements de ces derniers.

De son côté, les outils de l'économie comportementale peuvent également éclairer le paradoxe précédent. En s'appuyant sur la Prospect Theory de Kahneman et Tversky, on peut montrer qu'il n'y a aucune raison pour que la perception des risques par les acteurs corresponde toujours aux probabilités objectives des événements. Plus précisément, la Prospect Theory pose que les acteurs surpondèrent les petites probabilités et sous-estiment les fortes probabilités. L'une des conséquences les plus intéressantes de cette représentation de la décision en incertitude est le fait que les acteurs surréagissent aux variations des petites probabilités par rapport aux variations de probabilités plus élevées. Autrement dit, le fait que la fonction de transformation de probabilités ne soit pas linéaire permet d'expliquer que les agents ont des réactions différentes selon que c'est la probabilité de l'événement très peu probable qui se modifie ou la probabilité de l'événement plus probable. L'important dans la substitution entre motifs de licenciement ne serait pas alors la comparaison absolue des taux de recours mais le fait que le taux de recours concernant le licenciement économique, tout en étant très faible, 
est en constante augmentation(10), entraînant comme le prédit la Prospect Theory une surréaction des acteurs qui se détourneraient donc de plus en plus de ce motif de licenciement. Tout se passerait donc comme si les acteurs accordaient un poids plus important aux risques judiciaires du licenciement économique que sa réelle probabilité. Ceci pourrait notamment expliquer que, alors même que le taux de recours contre les licenciements économiques est extrêmement bas et que donc il est en fait une procédure de licenciement relativement sûre, le licenciement économique se trouve être finalement de moins en moins utilisé. Reste à comprendre d'où peut venir cette perception différenciée des probabilités par les acteurs économiques. Dans le cas du droit du travail, la raison doit en être recherchée dans les caractéristiques du droit et du processus judiciaires. Nous retrouvons ici une question qui traverse l'ensemble de l'économie du droit comportementale, à savoir l'incapacité où se trouve parfois le droit à lutter contre les biais cognitifs des agents (JOLLS, SunsteIn, 2006). On retrouve ici toute l'ambiguïté du droit comme objet social : à la fois vecteur d'information et structurant les anticipations des acteurs - et donc leur univers informationnel - tout en ne pouvant pas complètement jouer ce rôle et ce pour des raisons qui tiennent à sa nature même d'être une activité interprétative.

Enfin, une autre façon de tester la pertinence de la thèse de la substitution consiste à la soumettre à l'épreuve des faits. On l'a précédemment souligné, si l'on s'appuie sur un critère de rationalité simplifié, le différentiel en matière de taux de recours devrait plutôt exercer un effet désincitatif sur la probabilité de recourir à un LMP pour un employeur. Autrement dit, si substitution il y avait elle devrait s'exercer plutôt dans le sens opposé à celui évoqué par certains travaux. De manière complémentaire, on pourrait également considérer qu'un environnement judiciaire caractérisé par des risques de contentieux et des coûts judiciaires élevés devrait accentuer encore plus ce mécanisme de substitution inversée. Autrement dit, on pourrait s'attendre à ce que la part des LMP dans l'ensemble des licenciements soit d'autant plus faible, toute chose égale d'ailleurs, que les entreprises sont implantées dans des zones géographiques où les risques de contentieux et les coûts liés à ces contentieux sont élevés. Une exploitation des Déclarations de mouvements de main-d'ouvre (DMMO), qui renseigne sur les entrées et les sorties d'emploi parmi l'ensemble des établissements des cinquante salariés ou plus, croisées avec des données collectées au niveau des conseils des prud'hommes et des cours d'appel,

(10) La probabilité de recours en cas de licenciement économique quoique très faible n'a cessé d'augmenter pour passer de $1 \%$ en 1993 à 2,6\% en 2003 (Munoz-Perez, SerVerin, 2005). Nous sommes donc ici en présence d'une faible probabilité de recours mais en augmentation continue. permet de dégager une piste intéressante pour rendre compte de l'évolution contrastée des LMP et des LME. En effet, on peut observer, pour l'année 2005, que si le lien entre le contexte judiciaire local, défini par des variables de risque judiciaire et de coût judiciaire (11), et la part des LMP dans l'ensemble des licenciements est statistiquement non significatif en revanche le degré de tertiarisation du tissu productif est lié positivement à l'intensité du recours aux LMP. Ce résultat, s'il était confirmé par d'autres études, inviterait donc à rechercher une partie de l'explication de la montée de la part des LMP, non pas tellement du côté de l'utilisation stratégique du droit par les employeurs, mais plutôt dans la croissance du poids des services dans l'économie française.

\section{$*$ \\ $* \quad *$}

L'ambition qui a été la nôtre ici a été de montrer pourquoi il était intéressant de faire de l'analyse économique du droit du travail et de l'illustrer en prenant un fait stylisé bien connu des spécialistes des relations de travail. Au-delà de ce cas particulier, il nous semble important d'approfondir les interactions entre droit du travail et comportement économique. L'exemple ici développé renvoie à des outils spécifiques tels que la théorie des incitations, l'économie comportementale ou encore l'approche en théorie de la décision (psychologie du risque). Mais d'autres questions pourraient être posées, par exemple en étudiant les relations entre normes sociales et règles de droit. Il semble en effet que la définition des normes juridiques, par exemple le niveau de protection des salariés, puisse contribuer à déterminer les normes de comportements des agents. Par ailleurs, pour pouvoir appréhender l'influence du droit du travail sur les comportements des acteurs économiques et mieux rendre compte de la complexité des différents effets, il faudrait également pouvoir disposer de données de qualité sur le fonctionnement du marché du travail comme sur celui des juridictions impliquées dans les conten-

(11) Le risque judiciaire est mesuré, au niveau départemental, d'une part, par un indice de conflictualité, correspondant au rapport entre le nombre d'affaires nouvelles en procédure ordinaire dans les Conseils des prud'hommes du département, et le nombre total d'emplois salariés dans le département, et, d'autre part, par le taux de jugement, qui est obtenu en faisant le rapport entre le nombre total des affaires terminées en procédure ordinaire et le nombre total d'affaires nouvelles ordinaires dans les conseils de prud'hommes du département. Pour ce qui est du coût judiciaire, il est calculé, pour chaque département, par la durée moyenne des affaires terminées et par le taux d'appel, celui-ci étant obtenu en faisant le rapport entre le nombre d'affaires nouvelles provenant des prud'hommes des différents départements relevant de la cour d'appel considérée et le nombre d'affaires nouvelles en procédure ordinaire dans les conseils de prud'homme. 
tieux du travail. En effet, si la statistique publique permet de bien mesurer l'évolution des flux de licenciement, en revanche elle ne permet pas de quantifier précisément le nombre de licenciements effectués. De même, la statistique publique ne permet pas de mener une véritable analyse économique de l'évolution des contentieux du travail. En effet, construit pour répondre à des interrogations juridiques, le Répertoire général civil ne recense pas d'informations sur, par exemple, la taille des entre- prises impliquées dans le contentieux du travail, les caractéristiques socio-économiques des demandeurs (PCS, salaire antérieur, ancienneté, etc.), les prétentions des demandeurs ou les indemnités fixées en faveur des demandeurs. Favoriser la production de telles données permettrait certainement de favoriser le développement d'une analyse économique du droit du travail plus à même de tenir compte de la complexité des relations entre droit du travail et comportement économique.

\section{Bibliographie}

Arrunada B. (2007), "Pitfalls to Avoid when Measuring the Institutional Environment: Is 'Doing Business' Damaging Business?," Journal of Comparative Economics, 35, pp. 729-47.

Blanchard O., Tirole J. (2003), Protection de l'emploi et procédures de licenciement, rapport du Conseil d'analyse économique, Paris, La Documentation française, 76 pages.

Bourreau-Dubois C., Chaupain-Guillot S., Deffains B., Demougin D., Doriat-Duban M., Ferey S., Gabuthy Y., Guillot O., Jankeliowithch-Laval E., Lambert E.-A., Langlais E., RaY J.-C. (2008), "Analyse économique du droit du travail», rapport réalisé pour le compte de la DARES, 423 pages.

Cahuc P., Kramaz F. (2004), De la précarité à la mobilité: Vers une assurance sociale professionnelle, rapport au Ministre de l'emploi, du travail et de la cohésion sociale, décembre.

Cambdessus M. (2004), Le sursaut, vers une nouvelle croissance pour la France, collection «Rapports officiels», Paris, La Documentation française 202 pages.

Deffains B. (1997), «Analyse économique de la résolution des conflits juridiques», Revue française d'économie, vol. 12, n 3, pp. 57-99.

Deffains B. (2001), «Analyse économique de la responsabilité étendue en cas d'insolvabilité des pollueurs», Économie Publique, $\mathrm{n}^{\circ}$ 7, pp. 149-169.

Deffains B., Ferey S. (2007), «Théorie du droit et analyse économique», Droits, juillet, pp. 223-254.

Djankov S., La Porta R., Lopez-De Silanes F., Shleifer A., (2003), "Courts," Quarterly Journal of Economics, $118(2): 453-517$.

ICHINO A., RIPHALN (2003), “Are Judges biased by Labor Market Conditions?", European Economic Review, 47(5), pp. 913-944.

Jolls C., Sunstein C.R. (2006), "Debiasing Through Law,” Journal of Legal Studies, 35, pp. 199-241.
Kramars F., Michaud M.-L. (2004), “The Shape of hiring and separation costs", IZA Discussion Paper $\mathrm{N}^{\circ} 1170$, Institute for the Study of Labor.

Lagarenne C., Le Roux M. (2006), «Les licenciements en 2003 : trois fois plus nombreux pour motif personnel que pour raisons économiques", Premières Synthèses, DARES, mars, $\mathrm{n}^{\circ} 11.1,6$ pages.

Munoz-Perez B., Serverin E. (2005), Le droit du travail en perspective contentieuse 1993-2003, Ministère de la justice, cellule «Études et Recherches».

Pagano M., P. Volpin (2005), "Political Economy of Corporate Governance", American Economic Review, 10051030 .

Pignoni M.-T., Zouary P. (2003), «Les nouveaux usages du licenciement pour motif personnel», Premières Synthèses, DARES, $\mathrm{n}^{\circ} 28.2$, juillet, 8 pages.

Richet-Mastaing L. (2003), «Séries de données sur les mouvements de main-d'œuvre, 1996-2001», document d'étude, DARES, ${ }^{\circ} 72$, Juillet, Paris.

Roe M. (2007), Bankruptcy and Corporate Reorganization: Legal and Financial Materials (The Foundation Press 2d ed).

Tomasini M. (2003), «Depuis 10 ans, le turnover est en phase avec l'activité économique», Premières Synthèses, septembre, $\mathrm{n}^{\circ} 38$. DARES, 1, 6 pages.

Tomasini M., Le Roux M. (2002), «Diminution des licenciements économiques en $2000 »$, Premières Synthèses, mars, $\mathrm{n}^{\circ} 13.1$, DARES, 8 pages.

Tversky A., Kahneman D. (1992), “Advances in Prospect Theory: Cumulative Representation of Uncertainty", Journal of Risk and Uncertainty, 5, pp. 297-323.

De Virville M. (2004), Pour un Code du travail plus efficace, collection «Rapports officiels », Paris, La Documentation française, 97 pages.

Wasmer E. (2006), "The Economics of Prozac (Do Employees Really Gain from Employment Protection?)", Mimeo UQAM. 